\title{
MALNOURISHED OLDER ADULTS ADMITTED TO REHABILITATION IN RURAL NEW SOUTH WALES REMAIN MALNOURISHED THROUGHOUT REHABILITATION AND ONCE DISCHARGED BACK TO THE COMMUNITY: A PROSPECTIVE COHORT STUDY
}

\author{
S. Marshall ${ }^{1}$, A. Young'2, J. Bauer ${ }^{3}$, E. Isenring ${ }^{4}$
}

\begin{abstract}
Objectives: Understanding the nutritional journey that older adults make from rehabilitation to home will help to target nutrition screening and intervention programs. This study aimed to determine the nutritional status, physical function and healthrelated quality of life amongst malnourished older adults admitted to two rural rehabilitation units and 12 weeks post-discharge to the community. Design: Observational prospective cohort study, conducted August 2013 to February 2014. Setting: Rehabilitation units in rural New South Wales, Australia. Participants: Thirty community-dwelling, malnourished older adult inpatients (mean age $79.5 \pm 7.1$ years, $57 \%$ female). Intervention: Observation of usual care: basic nutrition services typical to rural rehabilitation units. Measurements: Outcome assessments were measured at rehabilitation admission, discharge and 12 weeks post-discharge, with nutrition status via the Scored Patient-Generated Subjective Global Assessment as the primary outcome measure. Secondary outcome measures included physical function (Modified Barthel Index) and health-related quality of life (Assessment of Quality of Life-6D). Results: At admission, half of the rehabilitation patients were moderately malnourished and half were severely malnourished, with the cohort becoming and remaining moderately malnourished on discharge and 12 weeks post-discharge. Only four patients $(24 \%)$ were well-nourished 12 weeks post-discharge. Following discharge, there was a trend showing decline in physical function. No improvement was found in health-related quality of life following discharge. Conclusion: Malnourished older adults admitted to rural rehabilitation units with basic nutrition care are likely to be discharged with moderate malnutrition, and remain moderately malnourished in the community for at least 12 weeks. Physical function and health-related quality of life remain poor in this population. Collaboration between health services and within the multidisciplinary team is essential to identify and treat malnourished older adults, and novel approaches for inpatient and post-discharge nutrition support is needed.
\end{abstract}

Key words: Rehabilitation, aged, malnutrition, community, nutrition status.

\section{Introduction}

The ageing population has caused a shift in the type of health care demanded, including an increased preference for independent living (1). Rehabilitation facilities play a vital role in increasing independence so that older adults with disability may return safely to the community. Rehabilitation is therefore likely to increase in importance

1. Faculty of Health Sciences and Medicine, Bond University; 2. Royal Brisbane and Women's Hospital, Herston, Queensland. Level 2 Dr James Mayne Building, Herston, Queensland, Australia; 3. Nutrition and Dietetics, School of Human Movement and Nutrition Sciences, Building 26, the University of Queensland, Brisbane, Queensland, Australia; 4. Nutrition and Dietetics, Faculty of Health Sciences and Medicine, Bond University. Bond Institute of Health and Sport, Robina, Queensland, Australia

Corresponding Author: Skye Marshall, Bond Institute of Health and Sport, Robina, Queensland, 4226, Australia. Telephone: +61 75595 5530, Fax: +61 755953524 , skye.marshall@student.bond.edu.au

Received March 23, 2015

Accepted for publication May 29, 2015 to the health care system as the proportion of older adults rises.

Malnutrition (undernutrition) is an expensive consequence and cause of disease. Between 30 and 60\% of rehabilitation older inpatients are malnourished, which presents a substantial economic and clinical challenge to rehabilitation facilities (2-4). A recent systematic literature review found that older adults admitted to rehabilitation with malnutrition had poorer health-related quality of life (HRQoL) and increased physical dysfunction, hospitalisation, institutionalisation and mortality once discharged to the community (4). However, no study has measured nutrition status in older adults following discharge from rehabilitation. It is therefore not known whether malnourished older adults are at risk of continued malnutrition once 
in the community (4). Understanding the nutritional journey older adults make from rehabilitation to home will help target nutrition screening and intervention programs. This is of particular importance in rural and remote Australia, due to the increased challenges in accessing, identifying and treating community-dwelling older adults with chronic disease (1). These challenges include a wide geographical spread, increased health care costs, limited health services, less availability of suitably qualified health professionals, less availability of informal care, and overall poorer health of the older adults (1). Therefore, this study aimed to determine the change in nutritional status, physical function and healthrelated quality of life amongst malnourished older adults admitted to two rural rehabilitation units and 12 weeks post-discharge to the community.

\section{Methods}

\section{Design}

This study was implemented as an observational prospective cohort study with data collected from August 2013 to February 2014.

\section{Participants}

Two public general rehabilitation units (24 and 31 beds) in the same local health district in rural New South Wales, Australia were chosen based on location. Participants were English-speaking inpatients $\geq 65$ years who were malnourished on admission (as assessed using the Scored Patient-Generated Subjective Global Assessment, PG-SGA (5)) and were chosen by consecutive sampling. Participants were eligible if they were community-dwelling prior to admission and had an informal caregiver. This includes community-dwelling patients transferred from acute care. Well nourished (Scored PG-SGA rating A) patients were excluded. The rehabilitation units do not admit patients with dementia.

\section{Routine clinical care}

Participants were placed on a high-protein highenergy (HPHE) food service diet code menu unless contraindicated by medical condition. The units each have approximately 0.15 full time equivalent (FTE, six hours per week) of dietetic services, significantly less than the recommended minimum of 1.0 and 1.25 FTE for units with 24 and 31 beds (6). Participants received individualised medical nutrition therapy by the rehabilitation dietitian only if referred by the rehabilitation team as part of usual care, which included nutrition screening on admission via the Malnutrition Screening Tool (7). Referrals were also made by the rehabilitation team if any nutritional problems became apparent to the team. Usual post-discharge nutrition support may include referral to publically-funded dietitian outpatient clinics, depending on individual patient needs and consent by the patient for the referral.

\section{Nutritional assessment}

The nutrition status of participants was assessed by the Scored PG-SGA (primary outcome measure) at admission (T1), discharge (T2) and 12 weeks post-discharge to the community (T3). If two data collection time-points occurred within six days, assessment of nutrition status was not repeated and it was assumed the nutrition assessment results had not changed in that short time period.

The Scored PG-SGA is a nutrition assessment tool that determines nutritional status based on medical history (weight change, dietary intake, symptoms that impact nutrition status and functional capacity) and physical examination (muscle and fat stores); and is sensitive to changes in nutrition status over a short period of time (8). It provides a continuous numerical score (with score of 7+ indicating malnutrition in older rehabilitation inpatients (9)), as well as a global rating of nutrition status for a nutritional diagnosis (" $\mathrm{A}$ " indicating "well nourished", "B" indicating 'moderate or suspected malnutrition', " $\mathrm{C}$ " indicating 'severe malnutrition') $(5,10)$. A higher numerical score indicates increased malnutrition/risk for malnutrition. A reduction in score on repeat measures indicates that nutrition status has improved. The Scored PG-SGA has shown strong concurrent and predictive validity in the geriatric rehabilitation setting (9).

Weight was measured using Tanita scales (BC-541, 2005, Tanita Corporation, Tokyo, Japan). If a participant was unable to stand unassisted then the rehabilitation ward chair or roll-on scales were used. All three scales were within $0.1 \mathrm{~kg}$ calibration. Weights reported for amputees were adjusted using standard algorithms (11, 12). Knee height was measured using a sliding knee height caliper and used to estimate height using a population specific formula $(13,14)$. BMI was calculated $\mathrm{kg} / \mathrm{m}^{2}$, and classifications for older adults used to determine underweight $\left(<23 \mathrm{~kg} / \mathrm{m}^{2}\right)$ and overweight/ obese $\left(>30 \mathrm{~kg} / \mathrm{m}^{2}\right)(15)$.

\section{Physical function and health-related quality of life}

The Modified Barthel Index (MBI) (16), a measure of physical function, and Assessment of Quality of Life (AQoL-6D) (17), a measure of HRQoL, were measured at discharge (T2) and 12 weeks post-discharge to the community (T3). The MBI (16) provides a numerical score (0-100, with 100 indicating total independence) as well as categories indicating dependency level (table 2) (16). The 
AQoL-6D is a multi-attribute assessment tool providing a numerical score (17). All outcome measurement tools were completed on behalf of the participant by the primary researcher, an Accredited Practising Dietitian, by verbal interview with the participant. Supplementary information was recorded from the patient's medical record, for example medications and list of comorbidities. Further clarification was sought from their informal caregiver or the rehabilitation staff if the participant's account was unreliable due to fatigue or limited shortterm recall following acute illness.

\section{Participant characteristics}

Participant descriptors and potential confounding variables, including age, gender, living arrangements, medical status and cultural background were identified from the participants' medical notes and self-reported by the participant. Cognitive impairment was assessed by occupational therapists as part of routine care.

\section{Statistical approach}

All statistical analysis was completed using SPSS version 22.0 [2013. IBM SPSS Statistics for Windows, Version 22.0. Armonk, NY: IBM Corp.]. Descriptive statistics were used to characterise participant descriptors and to report the outcome measures of the sample population (mean \pm SD/SE for normal variables, median (IQR) for skewed variables). Normality was assessed using the Shapiro-Wilk test. Change in nutrition status (Scored PG-SGA score) was determined by a linear mixed model to account for attrition and variation between the participants. The time-point was used as a repeated measures factor and the scaled identity as the covariance matrix of the random effect 'participant'. The analysis was carried out using the Restricted Maximum Likelihood method. Estimated Marginal Means were obtained, and pairwise comparisons using a Bonferroni adjustment were produced. The mean Scored PG-SGA score at each time-point was reported using these Estimated Marginal Means as opposed to observed means. Within-subject changes over time for nutrition status (Scored PG-SGA ratings) were examined between each of the three time-points and between the two timepoints (T2 and T3) for physical function and HRQoL. Continuous variables were assessed using the paired t-test, and categorical variables using the paired-samples sign test.

\section{Ethical consideration}

The MARRC (Malnutrition in the Rural Rehabilitation Community) Study has been was registered at the Australian New Zealand Clinical Trials Registry (ANZCTR: ACTRN12613000518763, Trial version
2.0, 10 July 2013) and has received ethical and governance approval (North Coast Human Research Ethics Committee approval number LNR 063, G108 and University of Queensland School of Human Movement Studies Ethics Committee approval number HMS13/0731). Written informed consent was obtained from all participants and/ or their guardians.

\section{Results}

\section{Sample population}

Thirty-one eligible patients were admitted during the recruitment period, of which 30 provided informed consent (response rate 97\%). Table 1 describes the characteristics of the participants in total and by rehabilitation site. Site A was found to have a significantly younger sample population, and a higher rate of admissions from the community than site $B$. Participant flow throughout the study is represented in Figure 1. Overall attrition was 43\%. Excluding three participants who had delayed discharge awaiting aged care placement, the length of stay ranged from 1-55 days, with a mean of $22.8 \pm 12.8$ days.

\section{Figure 1}

Patient flow through the three time-points: admission (T1), discharge (T2) and 12 weeks post-discharge (T3)

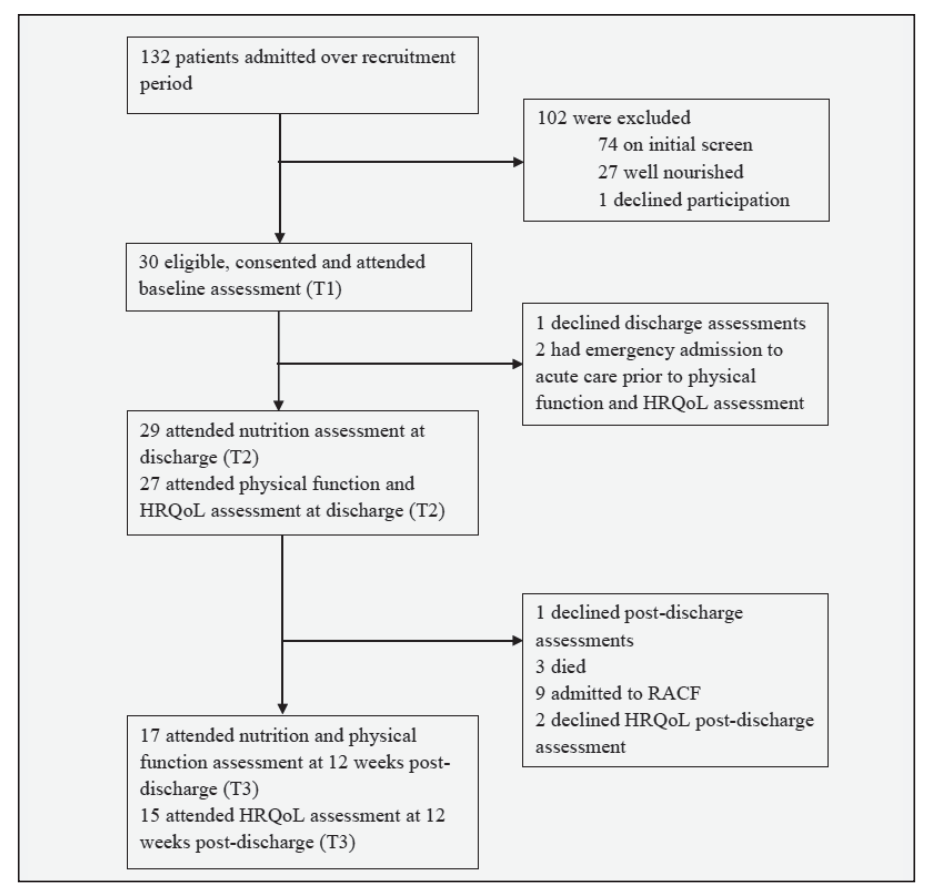

HRQoL, Health-related quality of life; RACF, Residential aged care facility

Twenty-three participants were placed on a HPHE diet code at admission; the main reason of contraindication was poorly-controlled diabetes. Approximately half $(n=16)$ of the participants were referred to the 
Table 1

Characteristics of participants by rehabilitation site and in total at admission

\begin{tabular}{|c|c|c|c|}
\hline Variable & Site A $(n=14)$ & Site $B(n=16)$ & Total participants $(n=30)$ \\
\hline Age (years, mean \pm SD) & $76.5 \pm 7.4$ & $82.1 \pm 5.9^{*}$ & $79.5 \pm 7.1$ \\
\hline Female $(\%)$ & 50.0 & 62.5 & 56.7 \\
\hline Weight (kg; median, IQR) & $55.5(46.1-67.8)$ & $58.1(49.8-65.8)$ & $56.2(47.9-65.2)$ \\
\hline BMI (kg/m²; median, IQR ) & $20.9(17.5-20.9)$ & $22.0(20.9-23.4)$ & $21.9(19.4-23.5)$ \\
\hline Underweight/BMI <23 (\%) & 64.371 .4 & 43.862 .5 & 66.753 .3 \\
\hline Overweight/obese/BMI >30 (\%) & 21.414 .3 & 12.56 .3 & 16.710 .0 \\
\hline \multicolumn{4}{|l|}{ Admission source: } \\
\hline - Acute health care facility (\%) & 71.4 & $93.8^{* *}$ & 86.7 \\
\hline - Community $(\%)$ & 28.6 & 6.3 & 13.3 \\
\hline \multicolumn{4}{|l|}{ Reason for admission: } \\
\hline - Acute illness (\%) & 64.3 & 100 & 80.0 \\
\hline - Chronic illness (\%) & 35.7 & 0.0 & 20.0 \\
\hline Living alone $(\%)$ & 7.1 & 25.0 & 16.7 \\
\hline \multicolumn{4}{|l|}{ Education level: } \\
\hline - Primary (\%) & 21.4 & 31.3 & 26.7 \\
\hline - Secondary $(\%)$ & 14.3 & 37.5 & 26.7 \\
\hline - Tertiary $(\%)$ & 57.1 & 6.3 & 3.3 \\
\hline - Trade $(\%)$ & 0.0 & 18.8 & 36.7 \\
\hline Polypharmacya (\%) & 100 & 93.8 & 96.7 \\
\hline \multicolumn{4}{|l|}{ Religion: } \\
\hline - Christian (\%) & 85.7 & 62.5 & 73.3 \\
\hline - No religion $(\%)$ & 14.3 & 25.0 & 20.0 \\
\hline - Other $(\%)$ & 0.0 & 6.3 & 3.3 \\
\hline \multicolumn{4}{|l|}{ Ethnicity: } \\
\hline - Aboriginal or Torres Strait Islander (\%) & 7.1 & 0.0 & 3.3 \\
\hline - Caucasian: European (\%) & 14.3 & 68.8 & 23.3 \\
\hline - Caucasian: Australasian (\%) & 78.6 & 31.3 & 73.3 \\
\hline - Other $(\%)$ & 0.0 & 0.0 & 0.0 \\
\hline \multicolumn{4}{|l|}{ Cognitive impairment ${ }^{\mathrm{b}}$ : } \\
\hline - MMSE (n=14) (mean \pm SD) & & $23.1 \pm 3.4$ & \\
\hline - 3MS Test $(n=20)($ mean $\pm S D)$ & $79.0 \pm 15.9$ & & \\
\hline Pensioner $(\%)$ & 85.7 & 81.3 & 83.3 \\
\hline Dentures $(\%)$ & 57.1 & 81.3 & 70.0 \\
\hline Domiciliary services at T4 & 3 & 4 & 7 \\
\hline Community nursing services at $\mathrm{T} 4$ & 2 & 0 & 2 \\
\hline
\end{tabular}

BMI, body mass index; IQR, interquartile range; SD, standard deviation. a. Considered $\geq 3$ prescribed medications at the time of nutrition assessment; $b$. Not compared between sites due to difference in measurement tools; * Significant difference between sites ( $P=0.028)$; ${ }^{* *}$ Significant difference between sites $(\mathrm{P}=0.022)$ 
rehabilitation dietitian; these participants had a range of one to four (median 1.0) appointments with the dietitian. No participants were referred by the rehabilitation team to see a community dietitian on discharge as part of usual care. Nine of the 17 participants who attended the 12 week post-discharge assessment (T3) consented to a community dietitian referral; however, no participants had attended an appointment by six months postdischarge although multiple appointment times were offered. Furthermore, only $12 \%$ of participants received community nursing services following discharge, and $41 \%$ received domiciliary services (assistance with activities of daily living). Participants who reported weight one month prior to admission $(\mathrm{n}=21)$ lost a mean $3.1 \pm 2.5 \mathrm{~kg}(4.8 \pm 4.1 \%$ body weight). Participants who reported weight six months prior to admission $(n=13)$ reported a loss of $10.1 \pm 4.4 \mathrm{~kg}(12.4 \pm 5.8 \%$ body weight).

\section{Figure 2}

Nutrition status of participants at each time point according to the Scored Patient-Generated Subjective Global Assessment (PG-SGA) ratings

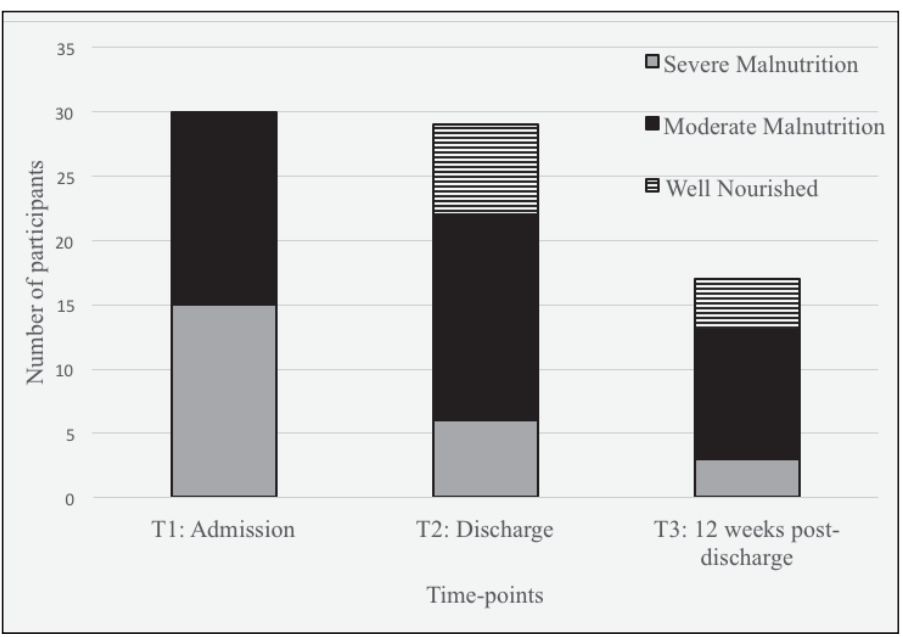

\section{Nutrition status}

The Scored PG-SGA scores and ratings, BMI and weight of participants at each of the time-points are presented in table 2. The Scored PG-SGA score and ratings were found to be significantly lower at $\mathrm{T} 2$ and T3 than at admission (T1) indicating an improvement in nutrition status during admission and post-discharge. However, according to the Scored PG-SGA global rating and score, the cohort remained malnourished at all time-points, with the mean score above the cut-off of 7. Post hoc analysis revealed the improvement in the Scored PG-SGA score following admission was due to weight stabilisation and some improvements in dietary intake, nutrition impact symptoms, physical function and medical status. When admitted to rehabilitation, $50 \%$ of the participants $(n=15)$ were rated 'moderately malnourished' and 50\% ( $\mathrm{n}=15)$ 'severely malnourished' according to the Scored PG-SGA. Throughout the study period the cohort became or remained 'moderately malnourished', where only four participants $(24 \%)$ were 'well-nourished' and three (18\%) 'severely malnourished' at 12 weeks post-discharge. Most of the improvement in nutrition status according to the Scored PG-SGA ratings was due to a participant improving from 'severely malnourished' to 'moderately malnourished' during admission. An equal number of participants improved, declined and had no change in nutrition status between discharge (T2) and 12 weeks post-discharge (T3), resulting in no change in nutrition status of the overall group from discharge to 12 weeks post-discharge. The trend towards moderate malnutrition is represented in figure 2. There was no change in BMI or body weight throughout the study period and the mean BMI remained "underweight" (BMI $<23 \mathrm{~kg} / \mathrm{m}^{2}$ ) at all time-points (table 2).

\section{Physical function and health-related quality of life}

The cohort had mild-moderate disability at discharge, and for those remaining in the study at 12 weeks there was no change in MBI score (table 2). Six of the eight participants scoring poorest in physical function (categories 1 - 3) at discharge did not attend follow-up at T3 due to admission to a residential aged care facility (RACF) or death. Categorically, a slight decline occurred in physical function, as only two participants improved in a category of physical function, nine had no change and six declined. No change was seen in HRQoL at 12 weeks post-discharge.

\section{Discussion}

This is the first study to measure the nutritional status of older adults following rehabilitation. Results suggest that the journey of the malnourished older adult from acute care, to rural rehabilitation facilities, to the community is bleak. Malnourished older adults admitted to rural rehabilitation units, whether severely or moderately malnourished, are likely to be discharged with moderate malnutrition, and remain moderately malnourished for at least 12 weeks in their homes. As patients were likely to be discharged with moderate malnutrition regardless of their length of stay, this suggests that the trend towards moderate malnutrition occurs early in the admission. Studies measuring nutrition status post-discharge from acute care facilities reported similar results in older adults $(18,19)$, indicating high risk for malnutrition following discharge from both acute and sub-acute health facilities.

The improvement in dietary intake and the decrease in nutrition impact symptoms prevented further weight loss from occurring; however the improvement was 
Table 2

The nutrition status, physical function and quality of life of older adults at admission, discharge and 12 weeks postdischarge

\begin{tabular}{|c|c|c|c|}
\hline & T1: Admission $(\mathrm{n}=30)$ & T2: Discharge $(n=29)$ & T3: 12 weeks post-discharge $(n=17)$ \\
\hline \multicolumn{4}{|l|}{ Adjusted Scored PG-SGA score } \\
\hline - mean $\pm S E$ & $11.2 \pm 0.7$ & $7.8 \pm 0.7^{*}$ & $8.3 \pm 0.9^{* *}$ \\
\hline - mean change from $\mathrm{T} 1$ & - & $-3.4^{*}$ & $-2.9^{* * *}$ \\
\hline - mean change from T2 & - & - & 0.5 \\
\hline \multicolumn{4}{|l|}{ Scored PG-SGA ratingb: } \\
\hline - A: Well-nourished & - & $7(24.1 \%)^{*}$ & $4(23.5 \%)^{* * * *}$ \\
\hline - B: Moderately malnourished & $15(50 \%)$ & $16(55.2 \%)^{*}$ & $10(58.8 \%)^{* * * *}$ \\
\hline - C: Severely malnourished & $15(50 \%)$ & $6(20.7 \%)^{*}$ & $3(17.6 \%)^{* * * *}$ \\
\hline $\mathrm{BMI} \mathrm{kg} / \mathrm{m}^{2}($ mean $\pm \mathrm{SD})$ & $22.3 \pm 4.3$ & $22.3 \pm 4.3$ & $22.0 \pm 4.5$ \\
\hline Weight kg (mean \pm SD) & $56.3 \pm 13.2$ & $56.4 \pm 12.6$ & $58.7 \pm 11.4$ \\
\hline MBI scorec (mean \pm SD) & - & $74.5 \pm 23.0 \mathrm{e}$ & $78.9 \pm 18.1$ \\
\hline \multicolumn{4}{|l|}{ MBI category b,c: } \\
\hline - 5: Minimal & & $8(29.6 \%) \mathrm{e}$ & $3(17.7 \%)$ \\
\hline - 4: Mild & & $11(40.7 \%)$ e & $11(64.7 \%)$ \\
\hline - 3: Moderate & & $4(14.8 \%) \mathrm{e}$ & $2(11.8 \%)$ \\
\hline - 2: Severe & & $2(7.4 \%) \mathrm{e}$ & 0 \\
\hline - 1: Total & & $2(7.4 \%) \mathrm{e}$ & $1(5.8 \%)$ \\
\hline AQoL-6D score $($ mean $\pm S D) d$ & - & $0.65 \pm 0.26^{\mathrm{e}}$ & $0.69 \pm 0.16^{\mathrm{f}}$ \\
\hline
\end{tabular}

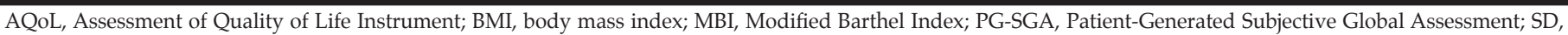

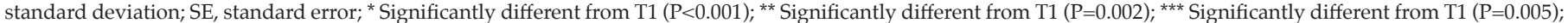

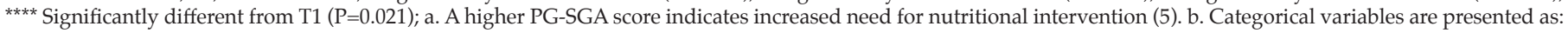

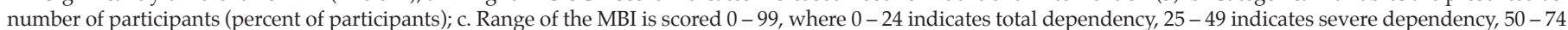

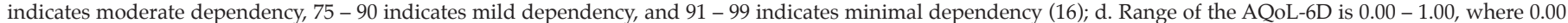

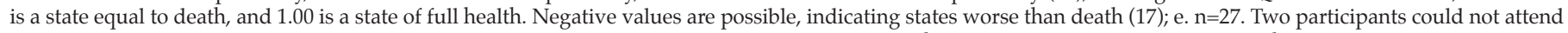
assessment due to emergency admission to acute care; f. $n=15$. Two participant's data excluded as the participants declined to complete the assessment forms.

not significant enough to allow patients to regain the weight, fat and muscle stores they lost prior to admission. In addition, these findings may represent a 'best case' scenario, as the cohort had informal caregivers to provide support with activities of daily living at home, and therefore may have better nutritional outcomes than those without this support (20). The poor rate of referral to the rehabilitation dietitian by the rehabilitation team reflects findings in previous studies, indicating that poor compliance with nutrition screening and referral is widespread and significant (21-23).

The small increase in MBI score between discharge and 12 weeks follow-up in the community appears to be skewed by attrition, where participants with the lowest scores and the greatest disability did not attend followup assessment due to admission to an RACF or death. Therefore the downward trend seen in the categories of physical function is likely to be clinically significant, and aligns with previous findings (24). Despite the slight improvement, this cohort still had a poorer MBI score post-discharge than a similar study in an Australian metropolitan rehabilitation unit (mean 78.9 versus 85.0 (24)). With continuing malnutrition and a downward trend physical function, it is no surprise that HRQoL remained lower than the Australian population norms for this age group $(\mu 0.65-0.69$ versus $\mu 0.75-0.77(25))$.

Post-discharge attrition reported by similar studies is substantially lower, ranging from $0-31 \%$ (4). However, these sample populations included well-nourished older adults, and therefore attrition due to death and RACF admissions would be expected to be lower. Alternatively, the higher rate of attrition in the current study may suggest that institutionalisation and mortality are higher in this rural cohort than metropolitan communities. A lower rate of attrition in similar studies is also due to differences in study design, where patients were included for follow-up assessment if they were in the community, in an RACF or had died (24, 26-28), whereas the current study attended follow-up assessment only on patients discharged to the community. Participants in the current study were only enrolled if they were admitted to rehabilitation with the view they would be discharged 
back to the community. The participants who had their discharge location changed from community to an RACF during admission were not excluded from the study and reported as attrition so that an accurate representation of the journey of community-dwelling malnourished older adults is reported.

\section{Research and practice implications}

From this study, it is clear that basic nutrition care with limited dietetic input during the inpatient rehabilitation admission is not sufficient to improve nutritional and functional status of malnourished older adults. Lack of referrals to the community dietitian on discharge, combined with poor attendance by participants referred at the conclusion of the study, highlights the need to review dietetic services and interventions during and after the rehabilitation setting.

Malnutrition is a significant and often silent contributor to 'post-hospital syndrome' which increases risk of rehospitalisation for conditions other than the original cause of admission (29). Therefore nutrition should be included in discharge summaries and handovers by medical, nursing and dietetic staff to ensure the continuum of care. Results suggest that early intervention is required in the geriatric rehabilitation setting. Advocacy by the multidisciplinary team for malnutrition to be of higher consideration on the rehabilitation agenda is called for.

The participants' informal caregivers were not involved in nutrition support in this study. The engagement of informal caregivers as part of the nutrition care team has been shown to be effective in improving the nutrition status of malnourished older adults in the community (30). Qualitative investigation is required to develop a patient-centred and informal caregiver-centred model of nutrition care for the rehabilitation setting. This model should be cost-effective, multidisciplinary and provide nutrition support during rehabilitation admission and post-discharge.

\section{Limitations}

A limitation of this paper is the small sample size which was related to a comparatively low patient turnover in rural rehabilitation units due to a longer length stay than in acute settings; however, the response rate was excellent in this at risk and hard to access population. In addition, results are of clinical significance and align with outcomes suggested by other studies in the geriatric rehabilitation setting which measured quality of life, physical function, health service use and mortality (4). The loss-to-follow-up 12 weeks postdischarge has been accounted for by the statistical approach, which lends confidence to the results.

\section{Conclusion}

Malnourished older adults admitted to rural rehabilitation units with basic nutrition care are likely to be discharged with moderate malnutrition, and remain moderately malnourished in the community for at least 12 weeks. Physical function and health-related quality of life remain poor in this population. Collaboration between health services and within the multidisciplinary team and sufficient dietetic services are essential to identify and treat malnourished older adults. Novel approaches for supporting patients and their informal caregivers during admission and post-discharge are needed. These results call for malnutrition to be of higher consideration on the rehabilitation agenda.

Acknowledgement: The authors gratefully acknowledge the assistance of E. Rathbone, Bond University, for contributing to the statistical approach and interpretation of data.

Conflict of interest: The authors declare they have no conflicts of interest. This study received no specific funding. SM is supported by an Australian Postgraduate Award throughout her PhD Candidature.

\section{References}

1. Caring for Older Australians, Volume 1. Canberra: Productivity Commission, 2011.

2. Watterson C, Fraser A, Banks M, Isenring E, Miller M, Silvester C et al Evidence based practice guidelines for the nutritional management of malnutrition in patients across the continuum of care. Nutrition \& Dietetics 2009;66:S1 - S34.

3. Elia M, Stratton R. The cost of disease-related malnutrition in the UK and economic considerations for the use of oral nutritional supplements (ONS) in adults. Redditch: BAPEN; 2005

4. Marshall S, Bauer J, Isenring E. The consequences of malnutrition following discharge from rehabilitation to the community: a systematic review of current evidence in older adults. J Hum Nutr Diet 2014;27:133-41.

5. Ottery FD. Patient-Generated Subjective Global Assessment. In: McCallum PD, Polisena CG, editors. The Clinical Guide to Oncology Nutrition. Chicago: The American Dietetic Association; 2000. p. 11-23.

6. Standards for the provision of inpatition adult rehabilitation medicine services in public and private hospitals Sydney, Australia: Australasian Faculty of Rehabilitation Medicine, The Royal Australasian College of Physicians, 2011.

7. Ferguson M, Capra S, Bauer J, Banks M (1999) Development of a valid and reliable malnutrition screening tool for adult acute hospital patients. Nutrition 1999;15:458-64.

8. Bauer I, Capra S, Ferguson M. Use of the scored Patient-Generated Subjective Global Assessment (PG-SGA) as a nutrition assessment tool in patients with cancer. Eur J Clin Nutr 2002;56:779-85.

9. Marshall S, Young A, Bauer J, Isenring E. Malnutrition in geriatric rehabilitation: prevalence, patient outcomes and criterion validity of the Scored Patient-Generated Subjective Global Assessment (PG-SGA) and the Mini Nutritional Assessment (MNA) Journal of the Academy of Nutrition and Dietetics In Press 2015.

10. Ottery FD. Rethinking nutritional support of the cancer patient: the new field of nutritional oncology. Semin Oncol 21, 1994.

11. Charney P, Malone A. Anthropometric assessment. ADA pocket guide to nutrition assessment, 2nd edition. Chicago, IL: American Dietetic Association; 2009. p. 160-1.

12. Osterkamp LK. Current perspective on assessment of human body proportions of relevance to amputees. J Am Diet Assoc 1995;95:215-8.

13. rxkinetics. Estimating height in bedridden patients Plattsburg, MO: rxkinetics; 1984-2013 [01/10/2013]. Available from: http:/ / www.rxkinetics.com/height_ estimate.html.

14. Nutrition screening as easy as mna. A guide to completing the Mini Nutritional Assessment (MNA). Swizterland Nestle Nutrition Institute.

15. Winter JE, MacInnis RJ, Wattanapenpaiboon N, Nowson CA. BMI and allcause mortality in older adults: A meta-analysis. The American Journal of Clinical Nutrition. 2014; DOI: 10.3945/ajcn.113.068122.

16. Shah S, Vanclay F, Cooper B. Improving the sensitivity of the Barthel Index for stroke rehabilitation. J Clin Epidemiol 1989;42:703-9.

17. Allen J, Inder KJ, Lewin TJ, Attia JR, Kelly BJ. Construct validity of the 
Assessment of Quality of Life-6D (AQoL-6D) in community samples. Health Qual Life Outcomes 2013;11:61.

18. Chen CCH, Tang ST, Wang C, Huang GH (2009) Trajectory and determinants of nutritional health in older patients during and six-month post hospitalisation. J Clin Nurs 2009;18:3299-307.

19. Young AM, Mudge AM, Banks MD, Rogers L, Allen J, Vogler B et al. From Hospital to Home: limited nutritional and functional recovery for older adults. Journal of Frailty and Aging In Press., 2015

20. Locher JL, Ritchie CS, Robinson CO, Roth DL, West DS, Burgio KL. A multidimensional approach to understanding under-eating in homebound older adults: The importance of social factors. Gerontologist 2008;48:223-34.

21. Raja R, Gibson S, Turner A, Winderlich J, Porter J, Cant R et al. Nurses' views and practices regarding use of validated nutrition screening tools. The Australian Journal of Advanced Nursing 2008;26:26.

22. Ross LJ, Mudge AM, Young AM, Banks M. Everyone's problem but nobody's job: staff perceptions and explanations for poor nutritional intake in older medical patients. Nutrition \& Dietetics 2011;68:41-6.

23. Lamb CA, Parr J, Lamb EI, Warren MD. Adult malnutrition screening, prevalence and management in a United Kingdom hospital: cross-sectional study. Br J Nutr 2009;102:571-5.

24. Neumann SA, Miller MD, Daniels L, Crotty M. Nutritional status and clinical outcomes of older patients in rehabilitation. J Hum Nutr Diet 2005;18:129-36.

25. Population norms for the AQoL-6D and AQoL-8D multi attribute instruments [Internet]. Centre for Health Economics, Monash University. [cited 02/07/2014].

26. Nicosia F, Bonometti F, Ghisla MK, Cossi S, Romanelli G, Marengoni A. Predictors of survival within 2 years of inpatient rehabilitation among older adults. European journal of internal medicine 2012;23:519-23.

27. Sullivan DH, Walls RC, Lipschitz DA. Protein-energy undernutrition and the risk of mortality within $1 \mathrm{y}$ of hospital discharge in a select population of geriatric rehabilitation patients. Am J Clin Nutr 1991;53:599-605.

28. Visvanathan R, Penhall R, Chapman I. Nutritional screening of older people in a sub-acute care facility in Australia and its relation to discharge outcomes. Age Ageing 2004;33:260-5.

29. Krumholz HM. Post-hospital syndrome-an acquired, transient condition of generalized risk. N Engl J Med 2013;368:100-2.

30. Marshall S, Bauer J, Capra S, Isenring E. Are informal carers and community care workers effective in managing malnutrition in the older adult community? A systematic review of current evidence. The Journal of Nutrition, Health \& Aging 2013;17:645-51. 